\title{
Tissue Kallikrein Exacerbating Sepsis-Induced Endothelial Hyperpermeability is Highly Predictive of Severity and Mortality in Sepsis
}

\author{
Xiao Ran' \\ Qin Zhang $\mathbb{D}^{2}$ \\ Shaoping $\mathrm{Li}^{1}$ \\ Zhen $\mathrm{Yu}^{\prime}$ \\ Li Wan ${ }^{2}$ \\ Bin $W^{3}$ \\ Rongxue $\mathrm{Wu}$ (ID $^{4}$ \\ Shusheng $\mathrm{Li}^{\prime}$ \\ 'Department of Emergency, Tongji \\ Hospital, Tongji Medical College, Huazhong \\ University of Science and Technology, \\ Wuhan, 430030, Hubei, People's Republic \\ of China; ${ }^{2}$ Department of Anesthesiology, \\ Tongji Hospital, Tongji Medical College, \\ Huazhong University of Science and \\ Technology, Wuhan, 430030, Hubei, \\ People's Republic of China; ${ }^{3}$ Laboratory of \\ Platelet and Endothelium Biology, \\ Department of Transfusion Medicine, \\ Wuhan Hospital of Traditional Chinese and \\ Western Medicine (Wuhan No.I Hospital), \\ Tongji Medical College, Huazhong \\ University of Science and Technology, \\ Wuhan, 430030, Hubei, People's Republic \\ of China; ${ }^{4}$ Department of Biological \\ Sciences Division/Cardiology, University of \\ Chicago, Chicago, IL, 60637, USA
}

Correspondence: Qin Zhang Email qzhang8@tjh.tjmu.cn

Shusheng Li

Email Shushengli16@sina.com

\begin{abstract}
Aim: Sepsis, an acute, life-threatening dysregulated response to infection, affects practically all aspects of endothelial function. Tissue kallikrein (TK) is a key enzyme in the kallikreinkinin system (KKS) which has been implicated in endothelial permeability. Thus, we aimed to establish a potentially novel association among TK, endothelial permeability, and sepsis demonstrated by clinical investigation and in vitro studies.
\end{abstract}

Methods: We performed a clinical investigation with the participation of a total of 76 controls, 42 systemic inflammatory response syndrome (SIRS) patients, and 150 patients with sepsis, who were followed-up for 28 days. Circulating TK levels were measured with an enzyme-linked immunosorbent assay. Then, the effect of TK on sepsis-induced endothelial hyperpermeability was evaluated by in vitro study.

Results: Data showed a gradual increase in TK level among controls and the patients with SIRS, sepsis, and septic shock $(0.288 \pm 0.097 \mathrm{mg} / 1$ vs $0.335 \pm 0.149$ vs $0.495 \pm 0.170$ vs 0.531 $\pm 0.188 \mathrm{mg} / \mathrm{l}$, respectively, $\mathrm{P}<0.001)$. Further analysis revealed that plasma TK level was positively associated with the severity and mortality of sepsis and negatively associated with event-free survival during 28 days of follow-up (relative risk, 3.333; 95\% CI, 2.255-4.925; $\mathrm{p}<0.001$ ). With a septic model of TK and kallistatin in vitro, we found that TK exacerbated sepsis-induced endothelial hyperpermeability by downregulating zonula occluden-1 (ZO-1) and vascular endothelial (VE)-cadherin, and these could be reversed by kallistatin, an inhibitor of TK.

Conclusion: TK can be used in the diagnosis of sepsis and assessment of severity and prognosis of disease. Inhibition of TK may be a novel therapeutic target for sepsis through increasing ZO-1 and VE-cadherin, as well as downregulating endothelial permeability.

Keywords: tissue kallikrein, sepsis, mortality, endothelial permeability, junction protein

\section{Introduction}

Sepsis, the leading cause of death in intensive care unit (ICU), is estimated to affect over 30 million people worldwide each year. ${ }^{1-3}$ Despite aggressive supportive treatment and prompt antibiotic administration, sepsis remains a life-threatening complication of infection, ${ }^{4,5}$ demonstrating that it had become an urgent task to search for a tool for the evaluation of the development and prognosis, in addition to explore new approaches for prevention and treatment of sepsis. ${ }^{6}$ Endothelial permeability is also thought to be a key factor in the progression from sepsis to septic shock. ${ }^{7-11}$ However, the underlying mechanism to induce hyperpermeability has not been well elucidated. 
Tissue kallikrein (TK) is a sort of key enzyme in the kallikrein-bradykinin system (KKS). ${ }^{12}$ As a serine protease, TK exerts a strong antihypertensive effect through the bradykinin receptor (BR) that is located in endothelium, ${ }^{13}$ indicating that TK may play a pivotal role in hemodynamic changes during the development of sepsis. It has also been proved that KKS is associated with vascular endothelial permeability, ${ }^{14}$ which plays a vital role in the pathogenesis of organ dysfunction in sepsis, ${ }^{10,11}$ while the role of TK, a key enzyme in tissue KKS, in the occurrence and development of sepsis was not clear. We, therefore, hypothesized that TK might be involved in the development of sepsis and septic shock through regulating endothelial permeability.

Endothelial barrier is composed of tight junctions (TJs) and adherens junctions (AJs). TJs are the main structural and functional molecules responsible for maintenance of the endothelial permeability barrier. ${ }^{15}$ Zonula occluden-1 (ZO-1) is a junctional adaptor protein that interacts with multiple junctional components ${ }^{16}$ and controls endothelial AJs and endothelial barrier formation. ${ }^{17}$ AJs play a role in maintaining the structural stability of TJs and are essentially composed of vascular endothelial (VE)cadherin. $^{18,19}$ Numerous studies have revealed that increasing of endothelial permeability decreased TJs and AJs in endothelial cells (ECs). ${ }^{20-22} \mathrm{We}$, therefore, supposed that elevated TK might upregulate vascular endothelial permeability by destroying TJs and AJs, and aggravate hypotension, and then lead to septic shock. To address the role of TK in sepsis, clinical research and in vivo study were performed. An observational study was undertaken to dynamically observe the changes of TK and indicate whether it can serve as a valid clinical indicator to predict the severity of sepsis and its prognosis. Then, we explored the effects of TK on endothelial permeability in the model of lipopolysaccharide (LPS)-induced sepsis in human umbilical vein endothelial cells (HUVECs) HUVECs.

\section{Materials and Methods Study Subjects}

All the patients with sepsis, septic shock, and systemic inflammatory response syndrome (SIRS) that met diagnostic criteria within 24 hours, who were admitted to ICU in 3 centers (Wuhan, China) from October 2014 to January 2015 and from June 2017 to September 2018, were enrolled into this study. The diagnostic criteria for SIRS, sepsis, and septic shock were defined and categorized as Sepsis-3 definitions described. ${ }^{6,23}$ The main exclusion criteria were systemic stress, SIRS status caused by simple trauma or surgery and patients who were admitted to the ICU several days after having been diagnosed with sepsis. Patients aged under 20 years or with acute cardiovascular events, such as coronary heart disease and stroke were excluded. Selected patients were clearly recorded with or without a history of chronic cardio-cerebrovascular diseases. In the same period, mild patients without obvious infection and systemic inflammatory response were assigned to control group. This study was conducted in line with the Declaration of Helsinki and was approved by the Ethics Committee of Tongji Hospital Affiliated to Tongji Medical College (TJ-IRB20150318). All the patients signed the written informed consent form prior to commencing the study.

\section{Follow-Up and Detection of Plasma TK Level}

Blood samples for TK measurements were taken within 24 $\mathrm{h}$ after clinical onset of sepsis on the ICU (day 1), as well as on days 3 and 7 of ICU hospitalization. All the patients with sepsis were followed-up to 28 days by phone after onset of disease. The main prognostic outcome was all-cause mortality after 28 days. Plasma TK level was measured with a biotin-avidin-amplified enzyme-linked immunosorbent assay (B-A ELISA) kit, which was designed as a solidphase, double-antibody, sandwich assay to detect plasma TK level. The detailed procedure had been described in our previous study ${ }^{24}$.

\section{In vitro Study}

HUVECs were purchased from the American Type Culture Collection (ATCC, Manassas, VA, USA) and cultured in Medium 199 (20\% fetal bovine serum (FBS), $0.1 \%$ glutamine, $0.01 \%$ heparin, $0.01 \% 50 \mathrm{mcg} / \mathrm{mL}$ endothelial cell growth supplement, $100 \mathrm{U} / \mathrm{mL}$ streptomycin and penicillin) at $37^{\circ} \mathrm{C}$ under $5 \% \mathrm{CO} 2$ with humidity of $95 \%$. The medium was partially changed every $48 \mathrm{~h}$ for 10-14 days till the cells grew to $60-70 \%$. Then, HUVECs were divided into the following four groups: (1) control: HUVECs were treated with phosphate-buffered saline (PBS); (2) Sepsis: HUVECs were stimulated by LPS $(100 \mu \mathrm{g} / \mathrm{mL})$ for $24 \mathrm{~h}$; (3) TK treatment: HUVECs were stimulated with LPS for $1 \mathrm{~h}$ and treated with human urinary kallidinogenase (HUK $1 \mathrm{uM}$ ) for $24 \mathrm{~h}$; (4) kallistatin 
treatment: HUVECs were stimulated with LPS for $1 \mathrm{~h}$ and treated with kallistatin $(160 \mathrm{ug} / \mathrm{mL})$ for $24 \mathrm{~h}$.

\section{Detection of Endothelial Permeability}

We used electric cell-substrate impedance sensing (ECIS) to detect endothelial permeability. That technique measures impedance of endothelial cells (Applied BioPhysics, Troy, NY, USA), as described in a previous study. $^{25}$ To measure impedance, cells were grown on 8W10E+ arrays (Applied BioPhysics, Inc., Troy, NY, USA). The arrays were treated with $10 \mathrm{mM}$ L-cysteine (cat\#C7352-25G; Sigma-Aldrich, St. Louis, MO, USA) followed by coating with type-I collagen (cat\#. A1048301, Thermo Fisher Scientific, Waltham, MA, USA). Then, electrical stabilization command in the ECIS was used. ECs were seeded onto the arrays at a density of 60,000 cells/cm2 in $400 \mu \mathrm{L}$ Dulbecco's modified Eagle's medium (DMEM). ECIS was conducted using the multiple frequency/time (MFT) to record the impedance measurements over a broad spectrum of frequencies.

\section{Western Blot Assay}

Protein lysates preparation and Western blots were undertaken as previously described. ${ }^{26}$ Total protein lysates were separated by $12 \%$ sodium dodecyl sulfate-polyacrylamide gel electrophoresis (SDS-PAGE) and transferred onto polyvinyl difluoride membranes (PVDF; Millipore, Bedford, MA, USA). Membranes were blocked with 5\% non-fat milk in TBST prior to incubating with anti-TK, anti-ZO-1 (Invitrogen, Carlsbad, CA, USA), and anti-VEcadherin (Invitrogen, Carlsbad, CA, USA) antibodies overnight at $4^{\circ} \mathrm{C}$. After extensive washing, membranes were incubated with horseradish peroxidase (HRP)conjugated secondary antibody for $1 \mathrm{~h}$ at room temperature. Images were obtained using a gel imaging system (BioRad Laboratories Inc., Hercules, CA, USA). Bands on blots corresponding to proteins of interest were analyzed by ImageJ software (NIH, Bethesda, MD, USA).

\section{Statistical Analysis}

Categorical variables were analyzed by chi-square test. Continuous variables were expressed as mean \pm standard deviation, and independent-samples $t$-test or one-way analysis of variance (ANOVA) was used to indicate statistical significance. Pearson's and Spearman correlation coefficients were used to test the correlation between continuous and categorical variables. Receiver operating characteristic
(ROC) curves were plotted to evaluate the diagnostic value of TK level and other traditional clinical indexes for sepsis. Unconditional logistic regression analysis was performed to evaluate the association of TK levels with sepsis. At last, we used Cox proportional hazards models and Kaplan-Meier survival estimates to assess the relationship between TK levels and 28-day survival in sepsis. SPSS 18.0 (IBM, Armonk, NY, USA) and MedCalc 15.8 (MedCalc Software Ltd., Ostend, Belgium) software were used for statistical analysis.

\section{Results \\ Baseline Characteristics of Study Population}

The present study eventually included 76 controls, 42 SIRS patients, and 150 patients with sepsis (62 and 88 patients with sepsis and septic shock, respectively). There were $36-62 \%$ male patients in each group, and 13-26\% with history of cardio-cerebrovascular diseases (CCVD). Lung was the most common source of infection with a proportion of $41-57 \%$, followed by abdominal infection accounted for 20-33\%. Compared with control and SIRS, the first-day clinical and laboratory indexes of patients with sepsis and septic shock had lower mean arterial pressure (MAP) and platelets, higher lactate, white blood cell (WBC) count, C-reactive protein (CRP), procalcitonin (PCT), interleukin (IL)-6 (IL-6), Acute Physiology and Chronic Health Evaluation II (APACHE II) score and Sequential Organ Failure Assessment (SOFA) score with a high 28-day mortality rate (Table 1).

\section{TK Level, Peaked on the First Day, Might Be a Promising Biomarker for Diagnosing Sepsis}

Both controls and SIRS patients were assigned to non-sepsis group, and plasma level of TK in patients with sepsis (sepsis and septic shock) after clinical onset from the first day to the seventh day was markedly higher than that in non-sepsis group though that was fluctuated on the third day $(0.516$ \pm 0.181 vs $0.461 \pm 0.205$ vs $0.488 \pm 0.147$ vs $0.305 \pm 0.119 \mathrm{mg}$ / 1, respectively, $\mathrm{P}<0.001$; Figure 1). ROC curves suggested that from the first day to the seventh day, plasma TK level had the most diagnostic value in the first day of patients with sepsis (Table 2 and Figure 2A-I). In comparison with traditional assessment indicators in the diagnosis of sepsis, TK was found more reliable than WBC count $(Z=5.893$, $\mathrm{P}<0.001)$, and similar with PCT $(\mathrm{Z}=1.188, \mathrm{P}=0.238)$, IL-6 
Table I Demographic and Clinical Characteristics of Study Population

\begin{tabular}{|c|c|c|c|c|c|}
\hline \multirow[t]{2}{*}{ Characteristics } & Controls & SIRS & Sepsis & Septic Shock & \multirow[t]{2}{*}{ P value ${ }^{A}$} \\
\hline & $(n=76)$ & $(n=42)$ & $(n=62)$ & $(n=88)$ & \\
\hline Male, n (\%) & 27 (36\%) & 26 (62\%) & $35(56 \%)$ & 48 (55\%) & $0.030^{*}$ \\
\hline Age, year & $38 \pm 14$ & $52 \pm 18$ & $53 \pm 17$ & $57 \pm 17$ & $<0.001 * *$ \\
\hline CCVD, n (\%) & $10(13 \%)$ & II (26\%) & 15 (24\%) & $18(20 \%)$ & $<0.001 * *$ \\
\hline $\begin{array}{l}\text { Infection site, } \mathrm{n}(\%) \\
\text { Lung } \\
\text { Abdomen } \\
\text { Urinary tract } \\
\text { Skin and soft tissue } \\
\text { Blood } \\
\text { Others }\end{array}$ & $\begin{array}{l}- \\
- \\
- \\
- \\
- \\
-\end{array}$ & $\begin{array}{c}24(57 \%) \\
8(20 \%) \\
3(8 \%) \\
3(6 \%) \\
3(6 \%) \\
1(3 \%)\end{array}$ & $\begin{array}{c}33(54 \%) \\
16(25 \%) \\
6(10 \%) \\
2(3 \%) \\
4(6 \%) \\
2(3 \%)\end{array}$ & $\begin{array}{c}36(41 \%) \\
29(33 \%) \\
13(15 \%) \\
2(2 \%) \\
1(1 \%) \\
6(7 \%)\end{array}$ & $\begin{array}{l}- \\
- \\
- \\
- \\
- \\
-\end{array}$ \\
\hline $\begin{array}{l}\text { Laboratory values } \\
\text { TK levels, } \mathrm{mg} / \mathrm{l} \\
\text { WBC, } 10^{9} / \mathrm{L} \\
\text { Platelets, } 10^{9} / \mathrm{L} \\
\text { Creatinine, umol/L } \\
\text { CRP, } \mathrm{mg} / \mathrm{L} \\
\mathrm{PCT}, \mathrm{ng} / \mathrm{mL} \\
\text { IL-6, } \mathrm{Pg} / \mathrm{mL} \\
\text { PO2/FiO2, } \mathrm{mmHg} \\
\text { Lactate, } \mathrm{mmol} / \mathrm{L}\end{array}$ & $\begin{array}{c}0.288 \pm 0.097 \\
9.7 \pm 3.5 \\
252 \pm 127 \\
75 \pm 50 \\
8.9 \pm 20.9 \\
1.6 \pm 9.2 \\
78 \pm 299 \\
- \\
1.909 \pm 0.757\end{array}$ & $\begin{array}{c}0.334 \pm 0.148 \\
13.8 \pm 7.1 \\
208 \pm 73 \\
250 \pm 377 \\
122 \pm 77 \\
13.2 \pm 19.2 \\
278 \pm 45 \mid \\
336 \pm 68 \\
2.118 \pm 0.824\end{array}$ & $\begin{array}{c}0.495 \pm 0.170 \\
16.2 \pm 8.1 \\
117 \pm 73 \\
225 \pm 247 \\
160 \pm 104 \\
32.4 \pm 36.9 \\
1247 \pm 1759 \\
224 \pm 56 \\
4.119 \pm 2.492\end{array}$ & $\begin{array}{c}0.531 \pm 0.188 \\
\mid 5.3 \pm 8.6 \\
113 \pm 9 \mid \\
214 \pm 140 \\
191 \pm 259 \\
47.5 \pm 38.7 \\
2111 \pm 1962 \\
198 \pm 48 \\
6.522 \pm 4.778\end{array}$ & $\begin{array}{l}<0.00 I^{* *} \\
<0.00 I^{* *} \\
<0.00 I^{* *} \\
<0.00 I^{* *} \\
<0.00 I^{* *} \\
<0.00 I^{* *} \\
<0.00 I^{* *} \\
\quad- \\
<0.00 I^{* *}\end{array}$ \\
\hline $\begin{array}{l}\text { ICU parameters } \\
\text { MAP, mmHg } \\
\text { ICU days } \\
\text { Catecholamine days } \\
\text { Ventilation days } \\
\text { CRRT days } \\
\text { APACHE II score } \\
\text { SOFA score } \\
\text { 28-days mortality, } \mathrm{n}(\%)\end{array}$ & $\begin{array}{c}89 \pm 16 \\
- \\
- \\
- \\
- \\
- \\
- \\
0(0)\end{array}$ & $\begin{array}{c}97 \pm 17 \\
8 \pm 3 \\
0 \pm 0 \\
2 \pm 1 \\
0 \pm 0 \\
7 \pm 4 \\
1 \pm 1 \\
8(19 \%)\end{array}$ & $\begin{array}{c}97 \pm 17 \\
7 \pm 5 \\
2 \pm 1 \\
5 \pm 2 \\
1 \pm 0.8 \\
18 \pm 6 \\
8 \pm 3 \\
30(48 \%)\end{array}$ & $\begin{array}{c}61 \pm 8 \\
12 \pm 6 \\
5 \pm 3 \\
7 \pm 5 \\
2 \pm 1 \\
26 \pm 8 \\
13 \pm 6 \\
61(69 \%)\end{array}$ & $\begin{array}{c}<0.00 I^{* *} \\
- \\
- \\
- \\
- \\
- \\
- \\
<0.001 * *\end{array}$ \\
\hline
\end{tabular}

Notes: Data are presented as $\mathrm{n}(\%)$ for categorical variables and mean \pm standard deviation of the mean (SD) for continuous variables with normal distributions. A groupwise comparison testing performed using $\chi 2$ test for categorical variables and One-way analysis of variance (ANOVA) for continuous variables with normal distributions. ${ }^{\mathrm{P}}$-values for the difference among controls, SIRS patients and patients with sepsis and septic shock, tested with one-way analysis of variance. $* \mathrm{P}<0.05$; $* * \mathrm{P}<0.0 \mathrm{I}$.

Abbreviations: CCVD, cardio-cerebrovascular disease; TK, tissue kallikrein; CRP, C-reactive protein; PCT, procalcitonin; IL-6, interleukin 6; PO2, partial pressure of O2; $\mathrm{FiO} 2$, fraction of inspiration $\mathrm{O} 2$; ICU, intensive care unit; MAP, mean arterial pressure; CRRT, continuous renal replacement therapy; APACHE, acute physiology and chronic health evaluation; SOFA, sequential organ failure assessment.

$(\mathrm{Z}=0.807, \mathrm{P}=0.419)$ and $\mathrm{CRP}(\mathrm{Z}=0.821, \mathrm{P}=0.412)$. A plasma

TK level of $0.340 \mathrm{mg} / \mathrm{l}$ had sensitivity of $88 \%$, specificity of 76.74\%, and Youden index of 0.647. When TK level was combined with PCT, the diagnostic value (area under the curve (AUC) $(95 \% \mathrm{CI})=0.948(0.921-0.967)$, sensitivity $=91.64 \%$, specificity $=89.15 \%$, Youden index $=0.808$ ) was more reliable $(\mathrm{Z}=5.516, \mathrm{P}<0.001)$. Therefore, detecting plasma TK level on the first day could be used independently or in combination with the PCT for the diagnosis of sepsis.

\section{Associations of TK Levels with Sepsis and Disease Severity}

Although correlations between TK level and general variables, such as age $(r=0.546 ; P=0.044)$ and MAP $(r=0.627$; $\mathrm{P}=0.016$ ) were confirmed, these were no longer significant in the multivariate linear regression model in controls. In septic patients, plasma TK level was positively correlated with ALT, AST and lactate, negatively correlated with PCT $(r=-0.176$; $\mathrm{P}=0.039)$ and MAP $(\mathrm{r}=-0.156 ; \mathrm{P}=0.007)($ Table 3$)$, and 


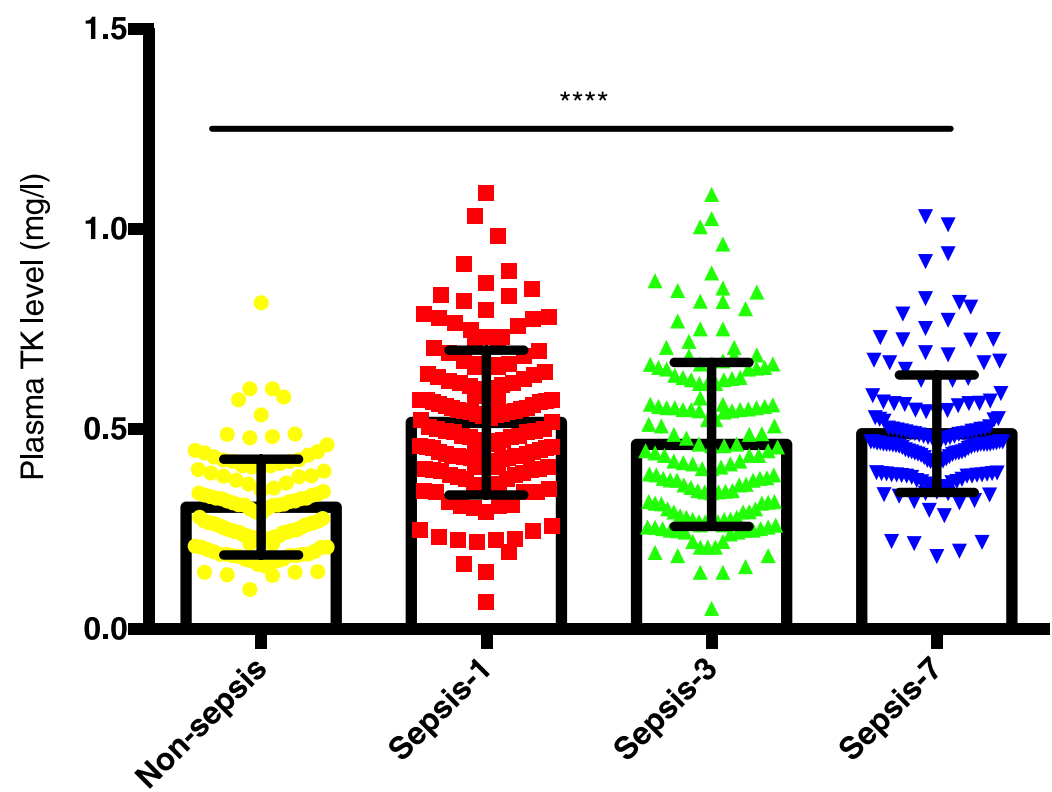

Figure I The mean plasma tissue kallikrein (TK) levels in controls and septic patients. TK levels in controls (76 controls and 42 SIRS patients) and septic patients who were admitted to ICU (62 septic patients and 88 patients with septic shock) on the day of diagnosis (Day I), 3 days after diagnosis (Day 3), and 7 days after diagnosis (Day 7 ) are displayed. Data were presented as mean \pm standard deviation of the mean (SD). One-way analysis of variance was used to indicate statistical difference. $* * * * P<0.000 \mathrm{I}$.

these associations were not apparent in multivariate linear regression model except for $\mathrm{PCT}(\mathrm{T}=-2.176 ; \mathrm{P}=0.032)$, ALT $(\mathrm{T}=-2.083 ; \mathrm{P}=0.040)$, and AST $(\mathrm{T}=3.156 ; \mathrm{P}=0.002)$. Logistic regression analysis showed a positive relationship between TK level and risk of sepsis (odds ratio (OR), 2.428; 95\% confidence interval (CI), 1.447-4.073; $\mathrm{P}=0.001)$.

All the patients are divided into 4 groups (control, SIRS, sepsis and septic shock) according to diagnostic criteria. A gradual increase in plasma TK level was observed among controls and patients with SIRS, sepsis, and septic shock $(0.288 \pm 0.097 \mathrm{mg} / \mathrm{l}$ vs $0.334 \pm 0.148$ vs $0.495 \pm 0.170$ vs 0.531 $\pm 0.188 \mathrm{mg} / \mathrm{l}$, respectively, $\mathrm{P}<0.001$; Table 1 and Figure $3 \mathrm{~A}$ ). Moreover, TK level was closely associated with the SOFA score $(\mathrm{R}=0.311, \mathrm{P}<0.001$; Figure $3 \mathrm{~B})$ and APACHE II score $(\mathrm{R}=0.344, \mathrm{P}<0.001$; Figure $3 \mathrm{C})$ in septic patients. Thus, plasma TK level could be used to assess the severity of sepsis.

\section{TK Levels Predicted Follow-Up Outcomes in Sepsis}

Among the 150 septic patients enrolled were 52 deaths for a 35\% all-cause 28-day mortality rate. They had markedly higher plasma TK levels than septic patients with survivor $(0.679 \pm 0.156$ vs $0.428 \pm 0.127, \mathrm{P}<0.001)$. We analyzed the association of TK levels with the risk of death in sepsis after adjusting for age, sex, SOFA score, WBC, CRP, PCT, and IL-6. The result showed that plasma TK level was positively associated with the risk of death in sepsis (Relative Risk (RR), 3.333; 95\% CI, 2.255-4.925; p < 0.001). Compared with the first quartile of plasma TK levels, the RR for death in the second quartile was 3.427 (95\% CI, 0.928-12.661; $\mathrm{p}=0.065)$. In the third and fourth quartiles, RRs were $4.517 \quad(95 \%$ CI, 1.274-16.013; $\mathrm{p}=0.020)$ and $28.944(95 \% \mathrm{CI}, 8.524-98.284 ; \mathrm{p}<0.001)$,

Table 2 ROC Curves in Assessing the Statue of Sepsis from the Ist Day to 7th Day

\begin{tabular}{|l|c|c|c|c|}
\hline Variables & Sensitivity (\%) & Specificity (\%) & AUC (95\% CI) & Youden Index \\
\hline TK (Day I) & 88.00 & 76.74 & $0.878(0.842-0.908)$ & 0.647 \\
TK+PCT (Day I) & 91.64 & 89.15 & $0.948(0.921-0.967)$ & 0.808 \\
TK (Day 3) & 67.03 & 80.00 & $0.788(0.736-0.834)$ & 0.47 \\
TK+PCT (Day 3) & 80.00 & 79.41 & $0.863(0.817-0.902)$ & 0.594 \\
TK (Day 7) & 81.48 & 77.5 & $0.859(0.811-0.898)$ & 0.59 \\
TK+PCT (Day 7) & 85 & 81.72 & $0.901(0.847-0.94 I)$ & 0.667 \\
\hline
\end{tabular}

Abbreviations: AUC, area under the curve; TK, tissue kallikrein; PCT, procalcitonin; TK+PCT, combination of TK and PCT for assessment of sepsis; Day I, the first day after clinical onset of sepsis on the ICU; Day 3, 3 days after diagnosis; Day 7, 7 days after diagnosis. 
A

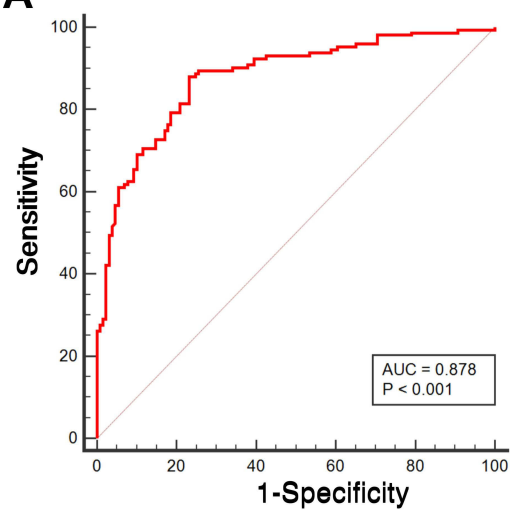

D

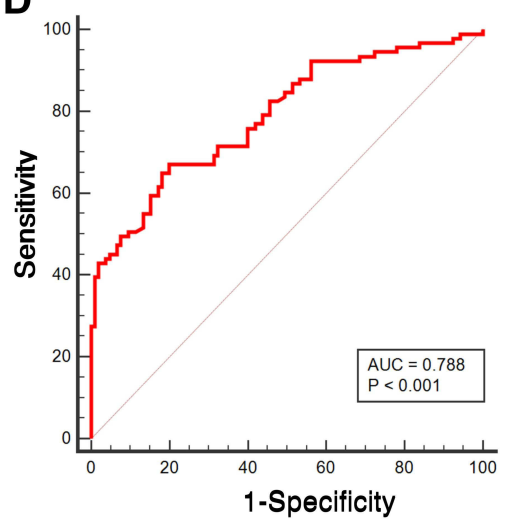

G

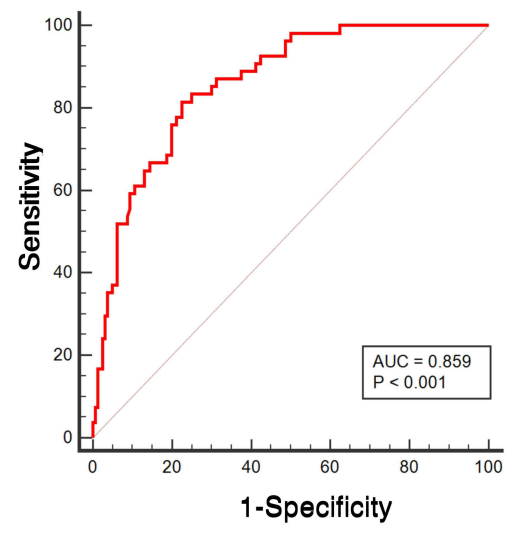

B

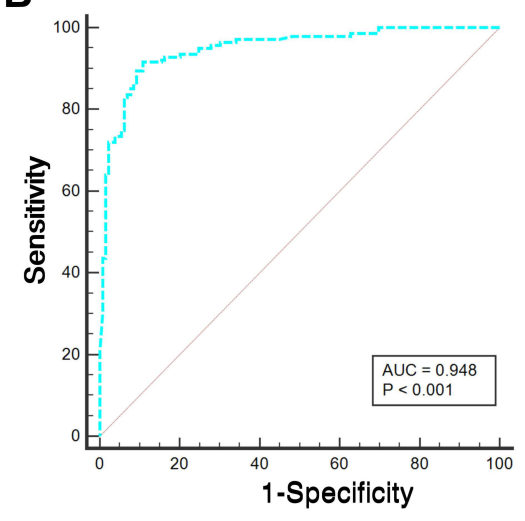

E

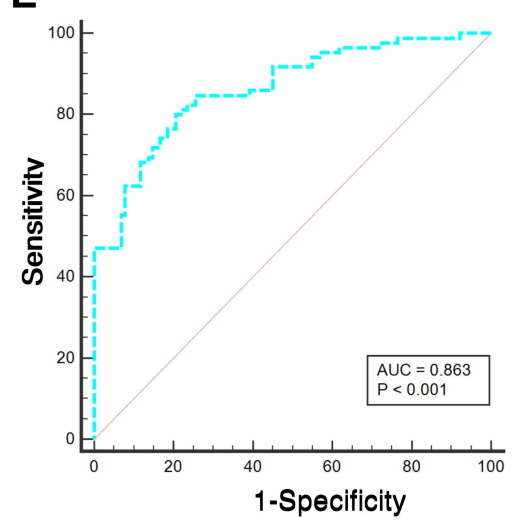

H

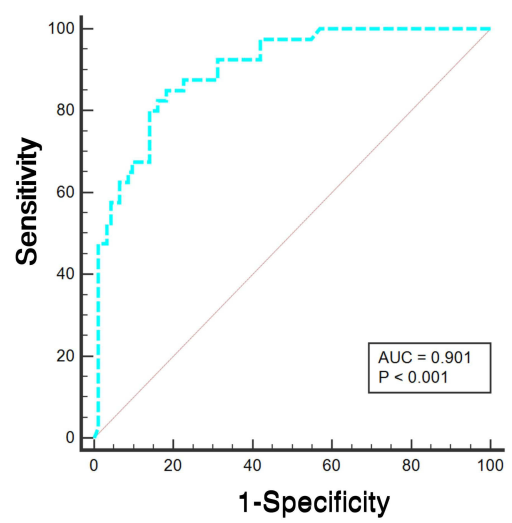

C

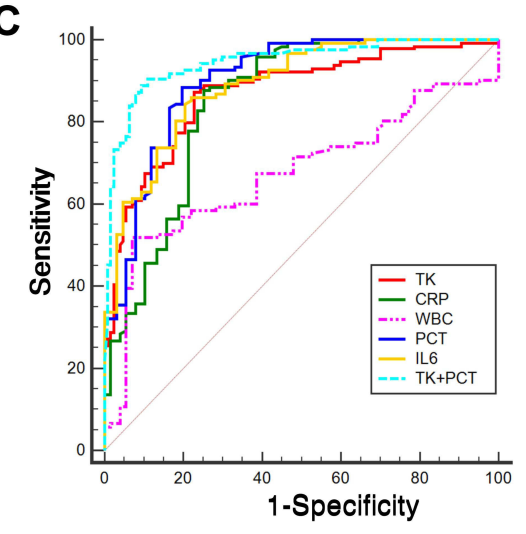

$\mathbf{F}$

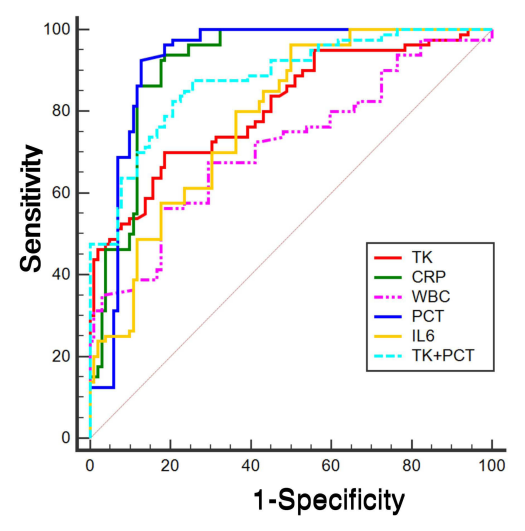

I

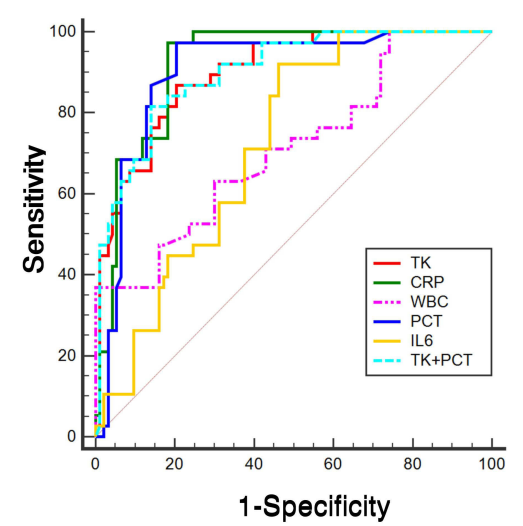

Figure 2 Diagnostic significance of TK on sepsis. ROC curves were plotted and pairwise comparisons were made among TK, CRP, WBC, PCT, IL6, and combination of TK and PCT for assessment of sepsis on the day of diagnosis (A-C), 3 days after diagnosis (D-F), and 7 days after diagnosis (G-I). Area under the curve (AUC) is shown in ( $\mathbf{A}$ and $\mathbf{B})$, (D and $\mathbf{E})$, and ( $\mathbf{G}$ and $\mathbf{H})$ groups.

respectively. Cox regression showed no significant association between traditional biomarkers (WBC, CRP, PCT, and IL-6) and the risk of death in sepsis. These suggest that only plasma TK level had the most prognostic value in sepsis. Further stratification analysis showed that there was no significant difference $(p=0.516)$ of prognostic values of
TK levels in sepsis patients (Relative Risk (RR), 6.431; 95\% CI, 2.252-18.368; $\mathrm{p}=0.001$ ) and septic shock patients (Relative Risk (RR), 3.573; 95\% CI, 2.102$6.071 ; \mathrm{p}<0.001)$. Kaplan-Meier survival estimates also indicated that compared with Quartile 1, Quartile 2 ( $24 \%, 26$ days vs $8 \%, 27$ days, Log rank test, $\mathrm{p}=0.079$ ); 
Table 3 Correlations Between TK Levels and General Variable in Septic Patients

\begin{tabular}{|l|c|c|}
\hline Variable & Correlations & P value \\
\hline Age & 0.152 & 0.071 \\
CRP & -0.038 & 0.673 \\
PCT & -0.176 & $0.039^{*}$ \\
IL-6 & 0.087 & 0.333 \\
WBC & 0.047 & 0.585 \\
MAP & -0.156 & $0.007^{* *}$ \\
HCT & -0.16 & 0.059 \\
Platelets & -0.06 & 0.482 \\
ALT & 0.181 & $0.032^{*}$ \\
AST & 0.281 & $<0.001^{* *}$ \\
Albumin & -0.021 & 0.808 \\
BUN & 0.022 & 0.799 \\
Creatinine & -0.115 & 0.177 \\
PO2 & -0.057 & 0.515 \\
Lactate & 0.202 & $0.018^{*}$ \\
\hline
\end{tabular}

Note: $* \mathrm{P}<0.05, * * \mathrm{P}<0.001$.

Abbreviations: CRP, C-reactive protein; PCT, procalcitonin; MAP, mean arterial pressure; HCT, hematocrit; ALT, alanine aminotransferase; AST, aspartate aminotransferase; BUN, blood urine nitrogen; PO2, partial pressure of O2; SOFA, sequential organ failure assessment; APACHE, acute physiology and chronic health evaluation.

Quartile 3 (33\%, 24 days vs 8\%, 27 days, Log rank test, $\mathrm{p}=0.008)$, and Quartile $4(89 \%, 11$ days vs $8 \%, 27$ days, Log rank test, $\mathrm{p}<0.001$ ) had higher risk of death and shorter event-free survival time (Figure 4). Therefore, elevated TK levels could be served as a predictor of outcome in sepsis.

\section{TK Exacerbated Sepsis-Induced Endothelial Hyperpermeability by Downregulating TJs and AJs}

The results of ECIS demonstrated that normalized transcellular electrical resistance (TER) was markedly decreased in sepsis group, and it appeared to be lower in the TK treatment group. However, this trend was reversed when HUVECs were treated with LPS and Kallistatin (Figure 5A and $\mathrm{B}$ ). These suggested that TK might enhance sepsis-mediated endothelial barrier dysfunction, and kallistatin could reverse this trend. Furthermore, in the sepsis group, the TK level was remarkably increased compared with that in the control group, and that was downregulated by kallistatin. In contrast, the protein expressions of ZO-1 and VE-cadherin were markedly decreased. These expressions were downregulated by TK and upregulated by kallistatin (Figure 5C-F), suggesting that the overexpression of TK was associated with the downregulation of ZO-1 and VE-cadherin in HUVECs. Collectively, decrease of TER and diminished expression of both ZO-1 and VE-cadherin revealed TK-mediated loss of HUVEC integrity.

\section{Discussion}

This is the first study that established a potentially novel link among TK, endothelial permeability, and sepsis demonstrated by clinical investigation and in vitro studies. We summarized the findings of the current study in Figure 6, which addressed evaluation values of TK on sepsis for the first time, and also assessed the role of TK in sepsis-mediated endothelial barrier disruption, indicating TK could be served as a valuable biomarker for diagnosis and assessment of disease severity and mortality of sepsis. Further, in vitro study suggested that inhibition of TK might be a therapeutic target for sepsis through increasing TJs and AJs, as well as downregulating endothelial permeability.

A strength of the present study is that we monitored plasma TK level after clinical onset of sepsis in the ICU (days 1, 3, and 7), and presented the evaluation values of TK on the early stage of sepsis. The data showed that from the first day to the seventh day, detecting plasma TK level in the first day had the most diagnostic value and could be used independently or in combination with the PCT as a strong diagnostic indicator of sepsis. This might be related to the massive depletion of TK during sepsis. ${ }^{27,28}$ Studies demonstrated that hemofiltration can significantly reduce various indicators of plasma infection. ${ }^{29}$ Hemofiltration therapy was applied to patients with sepsis in the current study, which might also interfere with plasma TK level. Therefore, detecting plasma TK level on the first day is more reliable in sepsis.

It is noteworthy that although CRP, PCT, and IL-6 levels play a similar role to plasma TK level in diagnosing sepsis, these traditional measures are closely associated with age, ${ }^{30}$ site of infection, and etiology of infection, ${ }^{31}$ while those are not conducive to predict the prognosis of patients with sepsis. Notably, compared with other testing indicators, plasma TK level was found more valuable in evaluation of the prognosis of sepsis. It was also reported as a robust indicator for assessment of the severity of sepsis. Collectively, plasma TK level could be a valuable biomarker for assessment of disease severity and mortality of sepsis.

The present study also explored the role of TK in sepsis-induced endothelial hyperpermeability. Our 
A

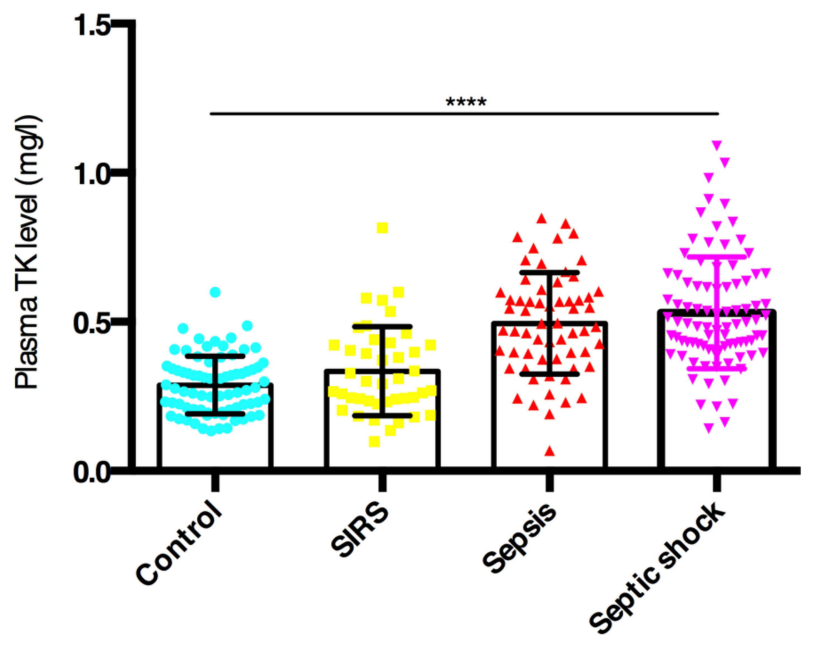

B

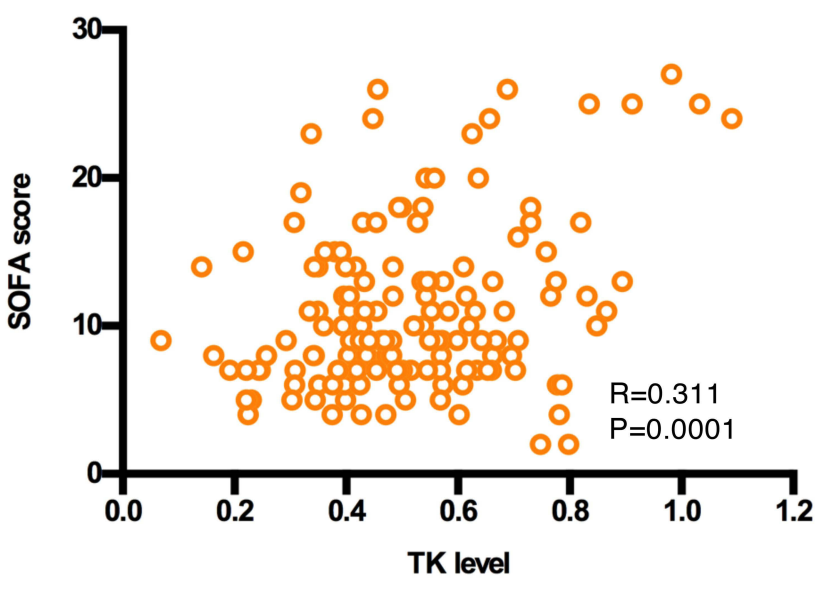

\section{C}

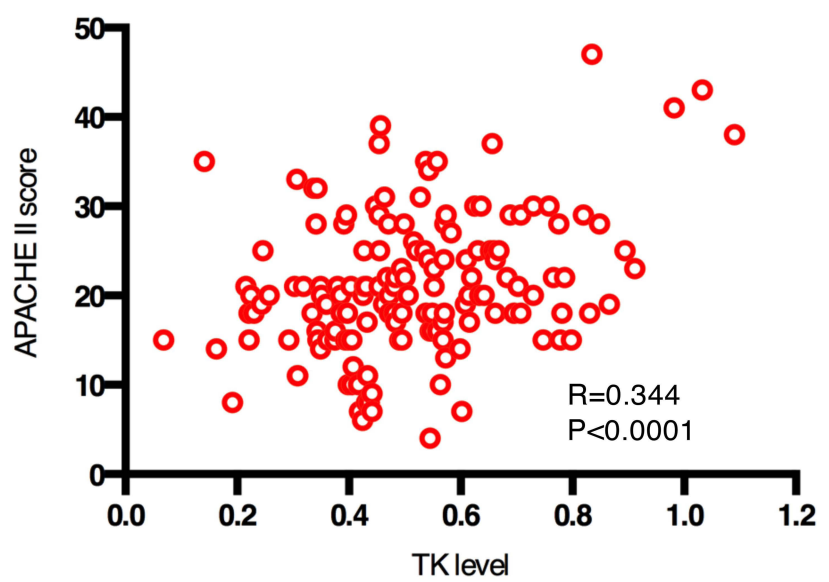

Figure 3 Tissue kallikrein (TK) levels predicted the severity of sepsis. (A) Mean plasma TK levels in controls and patients with SIRS, sepsis, and septic shock. Data are expressed as mean \pm standard deviation of the mean (SD). One-way analysis of variance was used to indicate statistical difference. $* * * * P<0.000$ I. (B) Correlation between TK levels and SOFA score. TK levels and SOFA score were highly correlative by bivariate correlation $(\mathrm{R}=0.3 \mathrm{II}, \mathrm{P}<0.00 \mathrm{I})$. (C) Correlation between TK levels and APACHE II score. TK levels and APACHE II score were highly correlative by bivariate correlation $(R=0.31 \mathrm{I}, \mathrm{P}<0.00 \mathrm{I})$.

previous study found that patients taking non-steroidal anti-inflammatory drugs (NSAIDs) had a higher level of $\mathrm{TK}^{24}$ Numerous studies confirmed that TK has antiinflammatory and multi-organ protective effects. ${ }^{13,32}$ However, plasma TK level was markedly increased in patients with sepsis in the current study. Is TK only a biomarker in the development of sepsis or is it involved in the development of sepsis? It has been proved that endothelial injury and hyperpermeability play a significant role in the development of sepsis. ${ }^{33}$ Sepsismediated endothelial dysfunction includes regulation of blood pressure, and inflammatory responses and barrier function of ECs. ${ }^{34}$ Capillary leak syndrome, one of the causes of high mortality, can exacerbate septic shock. The key mechanism causing capillary leak syndrome is to increase endothelial permeability. ${ }^{7,11,35}$ What is more, vascular hyperpermeability might depend on activation of the kallikrein-kinin system. ${ }^{14}$ These are consistent with our finding that TK could markedly enhance sepsis-mediated endothelial hyperpermeability and decrease TJs and AJs. Taken together, inhibition of TK might be an effective clinical therapy for managing sepsis and septic shock through downregulating TJs and AJs, as well as increasing endothelial permeability.

Numerous studies reported that a series of biological effects of TK is mediated by bradykinin through the 


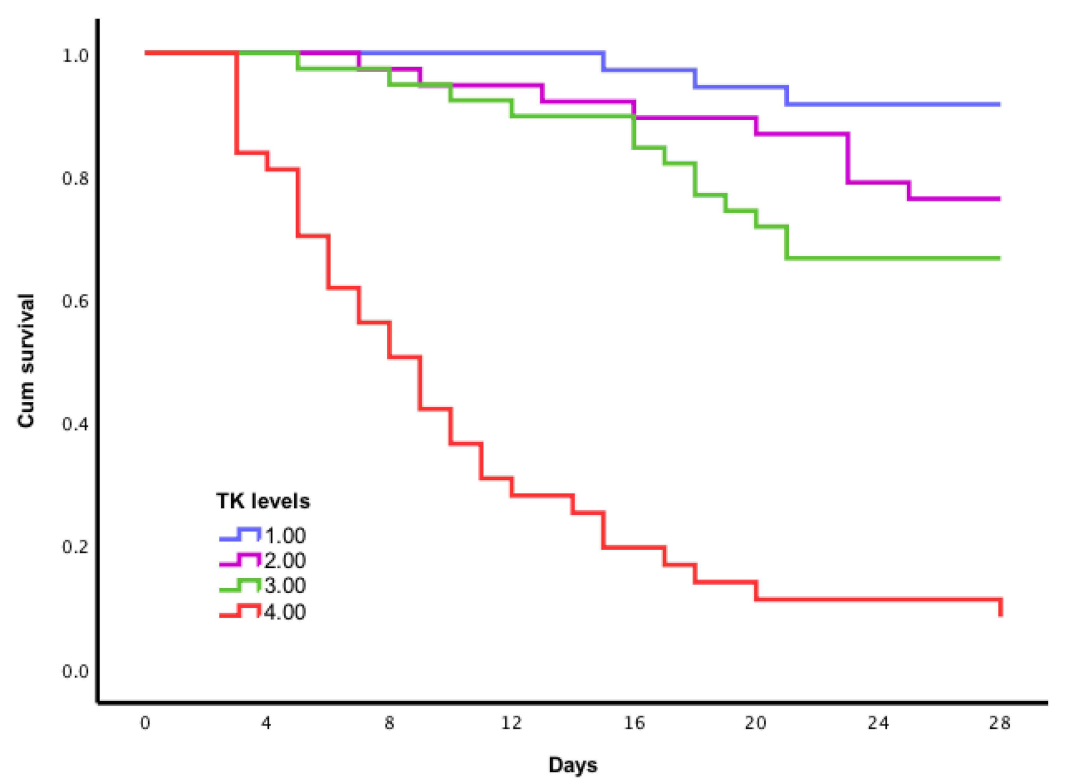

Figure 4 Kaplan-Meier survival curves based on plasma tissue kallikrein (TK) level. I = quartile I; 2 = quartile 2; $3=$ quartile 3; $4=$ quartile 4. Compared with Quartile I, Quartile 2 ( $24 \%, 26$ days vs $8 \%, 27$ days, Log rank test, $p=0.079)$; Quartile 3 (33\%, 24 days vs $8 \%, 27$ days, Log rank test, $p=0.008)$, and Quartile 4 ( $89 \%$, II days vs $8 \%, 27$ days, logrank test, $\mathrm{p}<0.00 \mathrm{I}$ ) had higher risk of death and shorter event-free survival time.

corresponding receptors (B1R and $\mathrm{B} 2 \mathrm{R})$. TK may induce anti-inflammatory, hypotensive, and organ protective effects through transient up-regulation of B2R in chronic cardiovascular and cerebrovascular diseases. However, with the development of inflammation, B1R may be activated continuously to produce significant hypotension or even shock and cause severe inflammatory reaction and tissue edema during the early stage of sepsis. $^{12,36,37}$ TK-specific inhibitor (Kallistatin) significantly controls the inflammatory response, ${ }^{38}$ improves hemodynamic deterioration and sepsis-induced multiple-organ dysfunction, ${ }^{39}$ suggesting that $\mathrm{TK}$ is not only associated with the development of inflammatory response to sepsis, but also with hemodynamic deterioration. We, therefore, suppose that TK-induced sepsis through two ways. One is that TK induces a dramatic reduction in blood pressure through B1Rmediated large amount of NO. Another is that TK exacerbates endothelial permeability and inflammation by activating B1R-mediated downregulation of $\mathrm{TJ} s$ and AJs.

Although the present study has a number of strengths, it still has several limitations as follows: First, according to the exclusion criteria, the sample size of the current study was relatively small. Second, sepsis is a highly heterogeneous disease. Patients with sepsis have complicated conditions and more confounding factors. Although various factors were taken in the analysis and assessment into account, it was still difficult to avoid the influence of confounding factors and the different clinical manifestation of septic patients. Third, TK levels were assessed postsepsis; therefore, we could not establish a causal link between elevated circulating levels of TK and sepsis. Fourth, other factors affecting endothelial permeability could not exclude. These can be better addressed by a large sample size study to dynamically evaluate the influence of TK on sepsis development and its prognosis and mechanism. Finally, the effect of TK on sepsisinduced endothelial hyperpermeability was only evaluated in the model of LPS-induced sepsis in HUVECs. Additional studies, including cecal ligation and puncture model in rodents may further elucidate whether TK contributes to sepsis-induced endothelial hyperpermeability.

\section{Conclusions}

In summary, the present study fills a previous gap by establishing a potentially novel link among TK, endothelial permeability, and sepsis, and revealed that TK could be used as a robust biomarker, as well as a therapeutic target for sepsis and septic shock. Taken together, these findings have substantial clinical and public health implications. Further extensive clinical studies on TK and sepsis are still required to confirm our findings. 
A

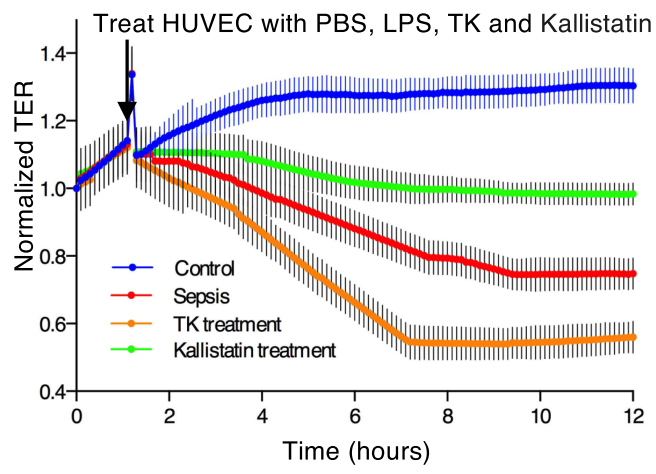

C

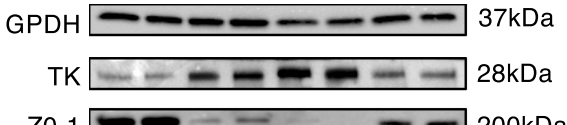

$\mathrm{ZO}-1 \longrightarrow 200 \mathrm{kDa}$

VE-cadherin $=120 \mathrm{kDa}$

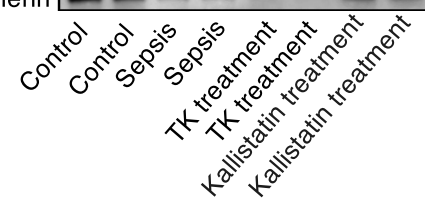

E

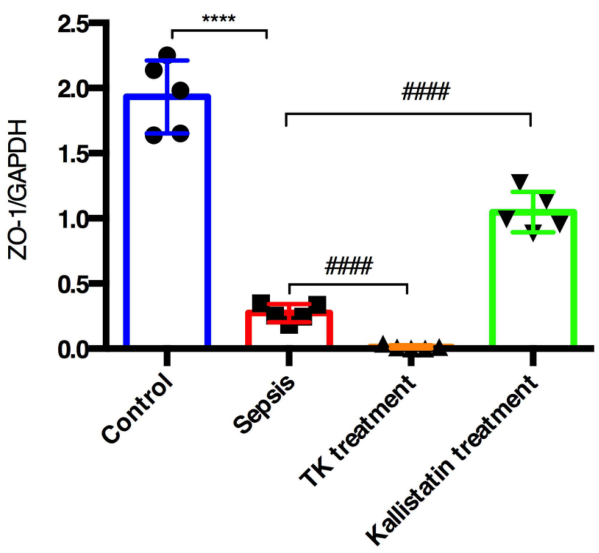

B

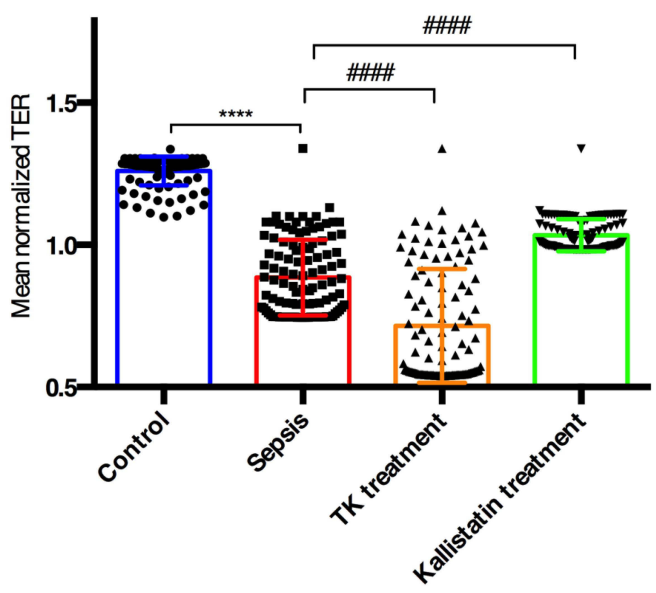

D

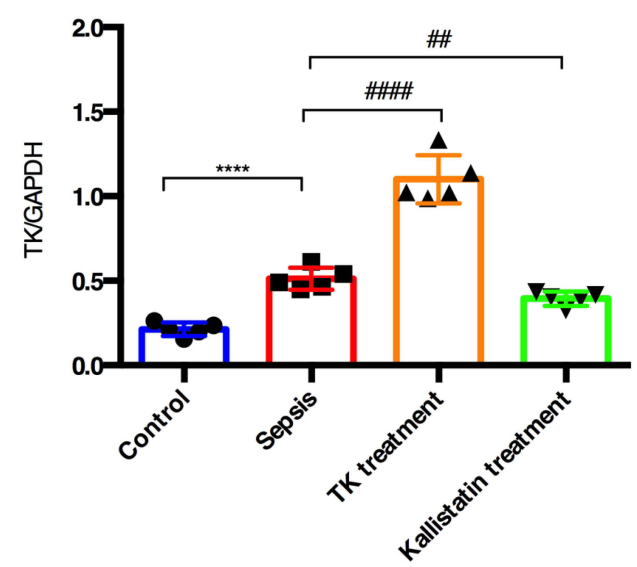

F

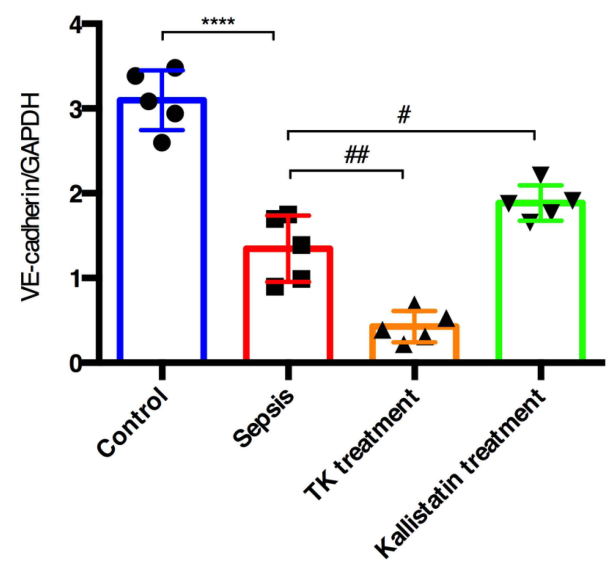

Figure 5 Tissue kallikrein (TK) exacerbated sepsis-induced endothelial hyperpermeability. TK treatment and Kallistatin treatment of HUVECs with LPS-induced sepsis model resulted in endothelial hyperpermeability (A) Real time electric cell-substrate impedance sensing (ECIS) measurements were conducted using normalized transcellular electrical resistance (TER) to record HUVECs permeability from 3 independent experiments. The lines denote the mean normalized resistance TERs \pm standard deviation of the mean (SD). (B) Mean normalized TERs were measured from 3 independent experiments using ECIS. ****P $<0.000 \mathrm{I}$ versus control; ${ }^{\# \# \# ~}<0.000$ I versus sepsis by two-tailed $t$-test. (C-F) Protein expressions of TK, ZO-I, and VE-cadherin were analyzed by Western blotting. Representative immunoblots were presented in the bar charts. The quantified OD of bands was corrected for the corresponding GAPDH. Data were presented as the mean \pm standard deviation of the mean (SD). $* * * * P$

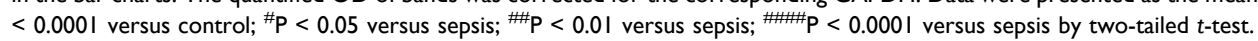




\section{Experimental Design}

\section{Clinical investigation}

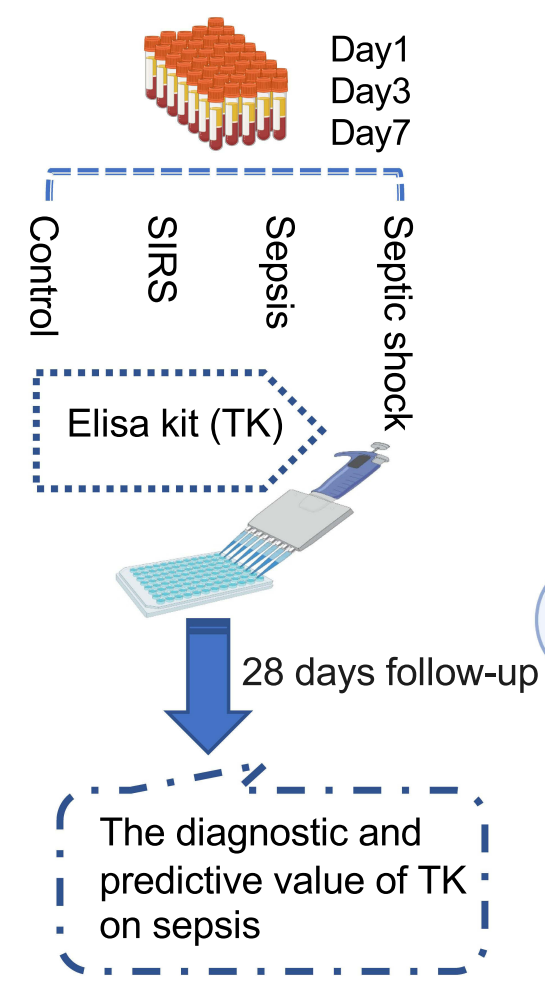

In vitro
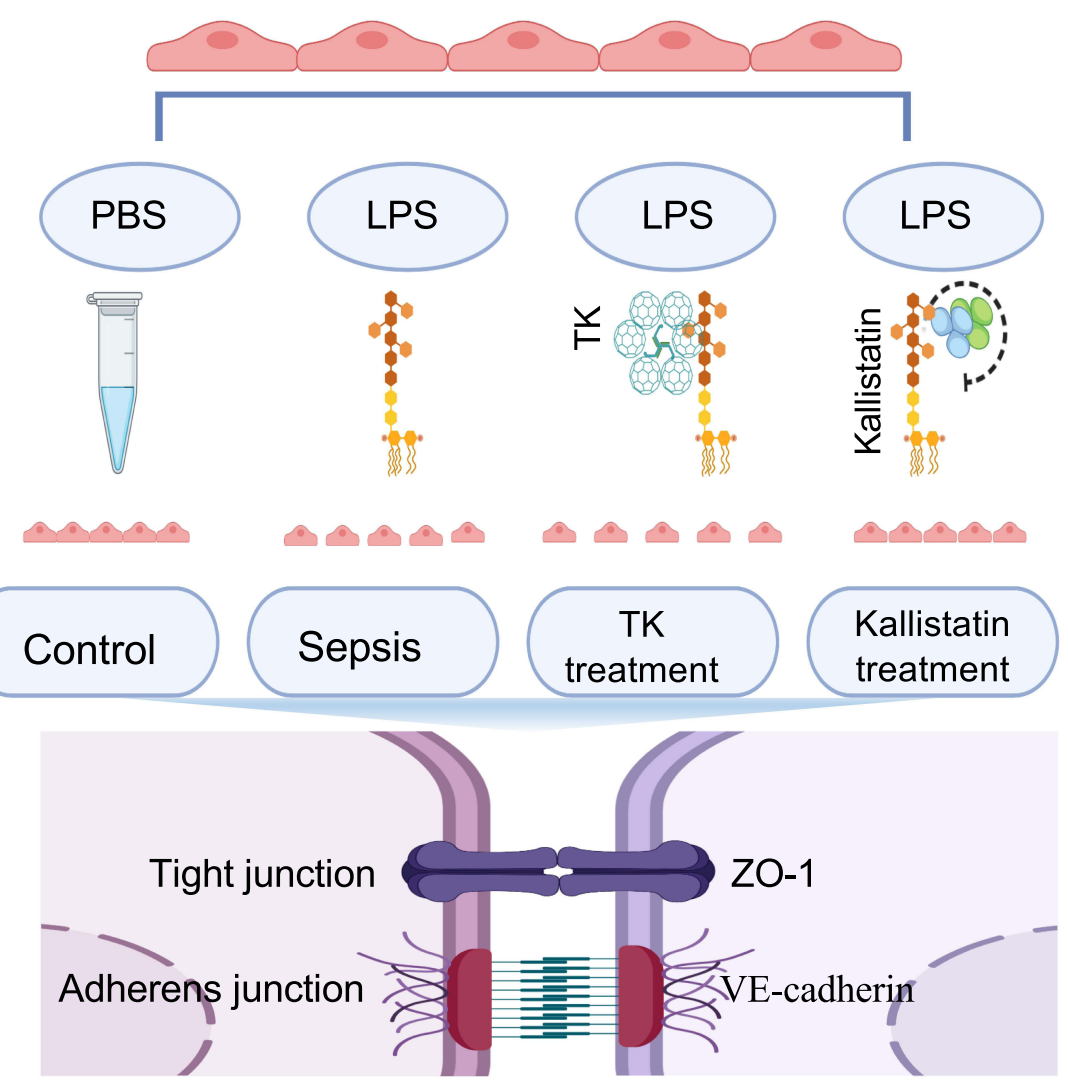

Figure 6 Experimental Design. Note: Created with BioRender.com.

\section{Abbreviations}

ICU, intensive care unit; TK, tissue kallikrein; KKS, kallikrein-bradykinin system; BR, bradykinin receptor; TJ, tight junction; AJ, adherens junction; ZO-1, Zonula occluden-1; VE, vascular endothelial; EC, endothelial cell; LPS, lipopolysaccharide; HUVEC, human umbilical vein endothelial cell; SIRS, systemic inflammatory response syndrome; B-A ELISA, biotin-avidin-amplified enzymelinked immunosorbent assay; FBS, fetal bovine serum; PBS, phosphate-buffered saline; HUK, human urinary kallidinogenase; ECIS, electric cell-substrate impedance sensing; MFT, multiple frequency/time; ANOVA, one-way analysis of variance; ROC, receiver operating characteristic; CCVD, cardio-cerebrovascular diseases; MAP, WBC, white blood cell; mean arterial pressure; CRP, C-reactive protein; PCT, procalcitonin; IL-6, interleukin (IL)-6; APACHE II, Acute Physiology and Chronic Health Evaluation II; SOFA, Sequential Organ Failure Assessment.

\section{Data Sharing Statement}

The data sets used and analyzed during the current study are available from the corresponding author on reasonable request.

\section{Acknowledgment}

TK was provided by Techpool Bio-Pharma Co., Ltd. (Guangzhou, China).

\section{Author Contributions}

All authors contributed to data analysis, drafting or revising the article, have agreed on the journal to which the article will be submitted, gave final approval of the version to be published, and agreed to be accountable for all aspects of the work.

\section{Funding}

Qin Zhang was supported by the National Natural Science Foundation of China (Grant No. 81100864). Xiao Ran is 
currently receiving a grant (No. 2019CFB730) from Science and Technology Plan Project of Hubei province, China.

\section{Disclosure}

The authors declare that they have no conflicts of interest for this work.

\section{References}

1. Dombrovskiy VY, Martin AA, Sunderram J, Paz HL. Rapid increase in hospitalization and mortality rates for severe sepsis in the United States: a trend analysis from 1993 to 2003. Crit Care Med. 2007;35 (5):1244-1250. doi:10.1097/01.CCM.0000261890.41311.E9

2. Fleischmann C, Scherag A, Adhikari NK, et al. Assessment of global incidence and mortality of hospital-treated sepsis. current estimates and limitations. Am J Respir Crit Care Med. 2016;193(3):259-272. doi:10.1164/rccm.201504-0781OC

3. Liu V, Escobar GJ, Greene JD, et al. Hospital deaths in patients with sepsis from 2 independent cohorts. JAMA. 2014;312(1):90-92. doi:10.1001/jama.2014.5804

4. Spatz M, Klatzo I. Pathological aspects of brain transport phenomena. Adv Exp Med Biol. 1976;69:479-495.

5. Meyer N, Harhay MO, Small DS, et al. Temporal trends in incidence, sepsis-related mortality, and hospital-based acute care after sepsis. Crit Care Med. 2018;46(3):354-360. doi:10.1097/ CCM.0000000000002872

6. Rhodes A, Evans LE, Alhazzani W, et al. Surviving sepsis campaign: international guidelines for management of sepsis and septic shock: 2016. Intensive Care Med. 2017;43(3):304-377.

7. Lee WL, Slutsky AS. Sepsis and endothelial permeability. $N$ Engl $J$ Med. 2010;363(7):689-691. doi:10.1056/NEJMcibr1007320

8. Kumar M, Leon Coria A, Cornick S, et al. Increased intestinal permeability exacerbates sepsis through reduced hepatic SCD-1 activity and dysregulated iron recycling. Nat Commun. 2020;11 (1):483. doi:10.1038/s41467-019-14182-2

9. Jorgensen VL, Nielsen SL, Espersen K, Perner A. Increased colorectal permeability in patients with severe sepsis and septic shock. Intensive Care Med. 2006;32(11):1790-1796. doi:10.1007/s00134-006-0356-6

10. Ince C, Mayeux PR, Nguyen T, et al. The endothelium in sepsis. Shock. 2016;45(3):259-270. doi:10.1097/SHK.0000000000000473

11. Druey KM, Greipp PR. Narrative review: the systemic capillary leak syndrome. Ann Intern Med. 2010;153(2):90-98. doi:10.7326/00034819-153-2-201007200-00005

12. Hillmeister P, Persson PB. The Kallikrein-Kinin system. Acta Physiol (Oxf). 2012;206(4):215-219. doi:10.1111/apha.12007

13. Regoli D, Rhaleb NE, Drapeau G, Dion S. Kinin receptor subtypes. J Cardiovasc Pharmacol. 1990;15(Suppl 6):S30-38. doi:10.1097/ 00005344-199015061-00007

14. Kobayashi H, Okada Y, Asahina T, Gotoh J, Terao T. The kallikrein-kinin system, but not vascular endothelial growth factor, plays a role in the increased vascular permeability associated with ovarian hyperstimulation syndrome. J Mol Endocrinol. 1998;20 (3):363-374. doi:10.1677/jme.0.0200363

15. Telo P, Lostaglio S, Dejana E. Structure of intercellular junctions in the endothelium. Therapie. 1997;52(5):395-398.

16. Umeda K, Ikenouchi J, Katahira-Tayama S, et al. ZO-1 and ZO-2 independently determine where claudins are polymerized in tight-junction strand formation. Cell. 2006;126(4):741-754. doi:10.1016/j. cell.2006.06.043

17. Tornavaca O, Chia M, Dufton N, et al. ZO-1 controls endothelial adherens junctions, cell-cell tension, angiogenesis, and barrier formation. J Cell Biol. 2015;208(6):821-838. doi:10.1083/ jcb.201404140
18. Dejana E, Tournier-Lasserve E, Weinstein BM. The control of vascular integrity by endothelial cell junctions: molecular basis and pathological implications. Dev Cell. 2009;16(2):209-221. doi:10.1016/j.devcel.2009.01.004

19. Shimizu F, Sano Y, Maeda T, et al. Peripheral nerve pericytes originating from the blood-nerve barrier expresses tight junctional molecules and transporters as barrier-forming cells. J Cell Physiol. 2008;217(2):388-399. doi:10.1002/jcp.21508

20. Chen JT, Lin YL, Chen TL, Tai YT, Chen CY, Chen RM. Ketamine alleviates bradykinin-induced disruption of the mouse cerebrovascular endothelial cell-constructed tight junction barrier via a calcium-mediated redistribution of occludin polymerization. Toxicology. 2016;368-369:142-151. doi:10.1016/j.tox.2016.09.004

21. Tian J, Shi R, Liu T, et al. Brain Infection by Hepatitis E virus probably via damage of the blood-brain barrier due to alterations of tight junction proteins. Front Cell Infect Microbiol. 2019;9:52. doi:10.3389/fcimb.2019.00052

22. Maherally Z, Fillmore HL, Tan SL, et al. Real-time acquisition of transendothelial electrical resistance in an all-human, in vitro, 3-dimensional, blood-brain barrier model exemplifies tight-junction integrity. FASEB J. 2018;32(1):168-182. doi:10.1096/fj.201700162R

23. Singer M, Deutschman CS, Seymour CW, et al. The Third international consensus definitions for sepsis and septic shock (Sepsis-3). JAMA. 2016;315(8):801-810. doi:10.1001/jama.2016.0287

24. Zhang Q, Ding H, Yan J, et al. Plasma tissue kallikrein level is negatively associated with incident and recurrent stroke: a multicenter case-control study in China. Ann Neurol. 2011;70 (2):265-273. doi:10.1002/ana.22404

25. Szulcek R, Bogaard HJ, van Nieuw Amerongen GP. Electric cell-substrate impedance sensing for the quantification of endothelial proliferation, barrier function, and motility. J Vis Exp. 2014;(85). doi: $10.3791 / 51300$.

26. Khalil H, Kanisicak O, Vagnozzi RJ, et al. Cell-specific ablation of Hsp47 defines the collagen-producing cells in the injured heart. $J C I$ Insight. 2019;4(15):e128722. doi:10.1172/jci.insight.128722

27. Smith-Erichsen N, Aasen AO, Gallimore MJ, Amundsen E. Studies of components of the coagulation systems in normal individuals and septic shock patients. Circ Shock. 1982;9(5):491-497.

28. Schmaier AH, Farber A, Schein R, Sprung C. Structural changes of plasma high molecular weight kininogen after in vitro activation and in sepsis. J Lab Clin Med. 1988;112(2):182-192.

29. Jing F, Wang J, Li M, et al. The influence of high volume hemofiltration on extra vascular lung water and alveolar-arterial oxygen pressure difference in patients with severe sepsis. Eur Rev Med Pharmacol Sci. 2015;19(20):3792-3800.

30. Lee WJ, Woo SH, Kim DH, et al. Are prognostic scores and biomarkers such as procalcitonin the appropriate prognostic precursors for elderly patients with sepsis in the emergency department? Aging Clin Exp Res. 2016;28(5):917-924. doi:10.1007/s40520-015-0500-7

31. Leli C, Ferranti M, Moretti A, Al Dhahab ZS, Cenci E, Mencacci A. Procalcitonin levels in gram-positive, gram-negative, and fungal bloodstream infections. Dis Markers. 2015;2015:701480. doi: $10.1155 / 2015 / 701480$

32. Spilberg I, Osterland CK. Anti-inflammatory effect of the trypsin-kallikrein inhibitor in acute arthritis induced by urate crystals in rabbits. J Lab Clin Med. 1970;76(3):472-479.

33. Kang Q, Chen Y, Zhang X, et al. Heat shock protein A12B protects against sepsis-induced impairment in vascular endothelial permeability. J Surg Res. 2016;202(1):87-94. doi:10.1016/j.jss.2015.12.034

34. Li Z, Yin M, Zhang H, et al. BMX represses thrombin-PAR1-mediated endothelial permeability and vascular leakage during early sepsis. Circ Res. 2020;126(4):471-485. doi:10.1161/CIRCRESAHA.119.315769

35. Yoo JW, Moon JY, Hong SB, Lim CM, Koh Y, Huh JW. Clinical significance of circulating endothelial cells in patients with severe sepsis or septic shock. Infect Dis (Lond). 2015;47(6):393-398. doi:10.3109/00365548.2014.1001999 
36. Gabra BH, Berthiaume N, Sirois P, Nantel F, Battistini B. The kinin system mediates hyperalgesia through the inducible bradykinin B1 receptor subtype: evidence in various experimental animal models of type 1 and type 2 diabetic neuropathy. Biol Chem. 2006;387 (2):127-143. doi:10.1515/BC.2006.018

37. Kuhr F, Lowry J, Zhang Y, Brovkovych V, Skidgel RA. Differential regulation of inducible and endothelial nitric oxide synthase by kinin B1 and B2 receptors. Neuropeptides. 2010;44(2):145-154. doi:10.1016/j.npep.2009.12.004
38. Griesbacher T, Rainer I, Tiran B, Evans DM. Involvement of tissue kallikrein but not plasma kallikrein in the development of symptoms mediated by endogenous kinins in acute pancreatitis in rats. $\mathrm{Br}$ J Pharmacol. 2002;137(5):692-700. doi:10.1038/sj.bjp.0704910

39. Li P, Guo Y, Bledsoe G, et al. Kallistatin treatment attenuates lethality and organ injury in mouse models of established sepsis. Crit Care. 2015;19(1):200. doi:10.1186/s13054-015-0919-4

\section{Publish your work in this journal}

The Journal of Inflammation Research is an international, peerreviewed open-access journal that welcomes laboratory and clinical findings on the molecular basis, cell biology and pharmacology of inflammation including original research, reviews, symposium reports, hypothesis formation and commentaries on: acute/chronic inflammation; mediators of inflammation; cellular processes; molecular mechanisms; pharmacology and novel anti-inflammatory drugs; clinical conditions involving inflammation. The manuscript management system is completely online and includes a very quick and fair peerreview system. Visit http://www.dovepress.com/testimonials.php to read real quotes from published authors.

Submit your manuscript here: https://www.dovepress.com/journal-of-inflammation-research-journal 